\title{
Los stents liberadores de everolimus podrían ser una alternativa a la cirugía de revascularización miocárdica en pacientes con compromiso de tronco de coronaria izquierda, sin alto riesgo
}

Everolimus-eluting stents could be an alternative to bypass surgery for non-high-risk patients with left main coronary artery disease

\section{Objetivos}

Determinar la no inferioridad del tratamiento de la enfermedad de tronco de coronaria izquierda $(\mathrm{TCl})$ con stents liberadores de droga (everolimus) comparado con la cirugía de revascularización miocárdica (CRM) en pacientes con puntaje de riesgo SYNTAX $\neq$ bajo o intermedio.

\section{Diseño, lugar y pacientes}

Ensayo clínico aleatorizado abierto, multicéntrico realizado en 126 centros en 17 países. Se estimó que una muestra de 1.900 pacientes otorgaría un poder de $80 \%$ para demostrar la no inferioridad de la angioplastia coronaria (ATC) en el punto final primario a 3 años con un margen de 4,2 puntos porcentuales. Se enrolaron 1.905 pacientes con enfermedad de $\mathrm{TCl}$ determinada por estenosis mayor de $70 \%$, o entre 50 y $69 \%$ con criterios funcionales (FFR) o imagenológicos (IVUS) de severidad.

\section{Intervención}

Los pacientes fueron aleatorizados a recibir ATC $(n=948)$ o
Stone GW y col. N Eng J Med. 2016;375(23):2223-35 CRM ( $n=957$ ) en bloques de 16, 24 o 32 y, estratificados según la presencia o ausencia de diabetes, y de acuerdo al puntaje SYNTAX mayor o menor de 23. Según el puntaje SYNTAX se clasificó a los pacientes en riego bajo ( 0 a 22), intermedio (23 a 32) o alto (mayor de 32).

\section{Medición de los resultados principales}

El resultado primario estuvo compuesto por muerte de cualquier causa, accidente cerebrovascular (ACV) o infarto a 3 los años de seguimiento. El punto final secundario fue un combinado de muerte, ACV o infarto a los 30 días, o muerte, ACV, infarto o necesidad de revascularización a los 3 años. El análisis se realizó por intención de tratar.

\section{Resultados principales}

A los 3 años de seguimiento, el punto final primario y los secundarios con ATC no mostraron inferioridad con respecto a la CRM (Ver Tabla 1).

Tabla 1. Resultados primarios y secundarios de ATC vs CRM en pacientes con enfermedad de TCI

\begin{tabular}{|c|c|c|c|c|}
\hline \multirow[b]{2}{*}{ Resultado } & ATC $(\mathrm{N}=948)$ & CRM (N = 957) & \multirow[b]{2}{*}{$\begin{array}{l}\text { Diferencia \% (por encima } \\
\text { del LS del IC) }\end{array}$} & \multirow[b]{2}{*}{ P (no inferioridad) } \\
\hline & Eventos (\%)* & Eventos (\%)* & & \\
\hline Primario (Muerte de cualquier causa, ACV o infarto a 3 años) & $137(15,4)$ & $135(14,7)$ & $0,7(4,0+)$ & 0,02 \\
\hline Secundario: Primario a 30 días & $46(4,9)$ & $75(7,9)$ & $-3,1(-1,2 \ddagger)$ & $<0,001$ \\
\hline Secundario: Primario + necesidad de revascularización a 3 años & $208(23,1)$ & $174(19,1)$ & $4,0(7,2 \ddagger)$ & 0,01 \\
\hline
\end{tabular}

ACV: Accidente cerebrovascular; ATC: angioplastia coronaria; CRM: cirugía de revascularización miocárdica; LS del IC: Límite superior del Intervalo de confianza; TCl: tronco de coronaria izquierda. * Tasa de eventos basadas en las estimaciones de Kaplan-Meier en los análisis de tiempo hasta el primer evento. † El valor representa el límite superior de confianza del 97,5\%. ‡ El valor representa el límite superior de confianza del $95 \%$.

\section{Conclusiones}

En el tratamiento de pacientes con enfermedad de tronco de coronaria izquierda y score SYNTAX bajo o intermedio, la angioplastia con stents liberadores de everolimus es no inferior a la CRM.

Fuente de financiamiento: Abbott Vascular

\section{Comentario}

El tratamiento de la enfermedad de $\mathrm{TCl}$ constituye un dilema constante en la práctica diaria. Varios intentos fallaron en demostrar beneficio de la ATC sobre la CRM siendo quizás el estudio SYN$\mathrm{TAX}^{2}$ la mayor referencia. El presente estudio de Stone y col. propone a la ATC como una alternativa segura, en el seguimiento a 3 años, en pacientes de riesgo intermedio y bajo (score SYNTAX menor de 33). La pequeña ventaja a favor del grupo ATC está dada por una mayor tasa de infarto en el grupo CRM. Sin embargo, no hay que perder de vista la mayor tasa de revascularización en el seguimiento del primer grupo $(12,6 \%$ vs $7,5 \%, p<0,001)$. Estrategias como la mayor utilización de imágenes en sala de hemodinamia (IVUS-OCT, utilizado en el $77 \%$ de los pacientes) podrían ayudar a disminuir este número. En el 2018 se publicó un meta-análisis ${ }^{3}$, que incorporó al presente estudio, y concluyó que la ATC es segura y factible a corto plazo; sin embargo, luego del año de seguimiento mostró mayor necesidad de revascularización y mayor número de infartos. Por este motivo, la ATC podría utilizarse en el tratamiento de la enfermedad de $\mathrm{TCl}$ en pacientes cuidadosamente seleccionados.

\section{Conclusiones del comentador}

Este importante ensayo clínico aleatorizado nos brinda información alentadora respecto a la seguridad de la angioplastia en enfermedad de $\mathrm{TCl}$. Llevado a la práctica diaria, debería permitir un crecimiento en la experiencia de los distintos centros, ayudando así a mejorar los resultados. Habría que aguardar los resultados a un plazo mayor a 3 años para poder trasladar estas recomendaciones a las guías de práctica clínica. Por último, la decisión de la estrategia terapéutica debería estar ligada a dos puntos importantes: la correcta evaluación del paciente por un equipo formado por un clínico, un intervencionista y un cirujano, y a los resultados obtenidos con ambas técnicas en cada centro.

Fernando Cohen [ Hemodinamia y Cardiología Intervencionista, Hospital Italiano de Buenos Aires. fernando.cohen@ hospitalitaliano.org.ar ]

Cohen F. Los stents liberadores de everolimus podrían ser una alternativa a la cirugía de revascularización miocárdica para pacientes con compromiso de tronco de coronaria izquierda, sin alto riesgo. Evid Actual Pract Ambul 2018;21(4):110. Comentado de: Stone GW, y col. Everolimus-Eluting Stents or Bypass Surgery for Left Main Coronary Artery Disease. N Eng J Med. 2016;375(23):2223-35. PMID: 27797291.

\section{Referencias}

1. SYNTAX score calculator (http://ir-nwr.ru/calculators/syntaxscore.htm).

2. Serruys PW, y col. Percutaneous coronary intervention versus coronary-artery bypass grafting for severe coronary artery disease. N Engl J Med. 2009 Mar 5;360(10):961-72.

3. Ali WE, y col. Meta-analysis study comparing percutaneous coronary intervention/drug eluting stent versus coronary artery bypass surgery of unprotected left main coronary artery disease. Clinical outcomes during short-term versus long-term (>1 year) follow-up. Medicine (Baltimore) 2018;97:7:e9909.

‡EI puntaje SYNTAX es un sistema de puntuación compuesto por variables derivadas de las características angiográficas coronarias. Combina la importancia del segmento coronario enfermo y las características angiográficas de la lesión. Se utiliza ante un paciente estable con enfermedad coronaria multivaso para decidir el tratamiento. 